The infection that did develop was never so severe as to warrant antibiotic treatment, and only local dressings were used to treat the infected wound.

Wound healing could be observed at any stage, as the dressing remained transparent throughout, and results revealed that 76 of the 108 cases $(70.4 \%)$ had complete healing of the wound by the fifth day. The life of the plastic dressing on parts exposed to water and minor trauma was found to be three days with a thin film used, and five days with a thicker film. Nine cases developed infection of the wound, and after treatment small areas of healing by second intention were present in the scar. This infection occurred in lacerated wounds only, all of which had presented with wounds contaminated by dirt and grit. None of the 48 minor surgical cases developed wound infection and all healed by first intention.

If perfect approximation of the skin edges was not obtained there was often haemorrhage from the wound when nobecutane dressing was applied. This complication occurred in 12 of the cases $(11.1 \%)$. As a result of experience, all cases with imperfect approximation of the skin edge and those who bled when the dressing was applied had a firm dry dressing for 12 to 24 hours. After this the dry dressing was removed and nobecutane substituted, without any haemorrhage occurring.

Suture removal never proved difficult, as use of the affected part usually resulted in much of the plastic dressing flaking off by the time the sutures were due to be removed.

\section{Illustrative Cases}

Case 1.-A slater and tiler doing a dirty job sustained a laceration of the dorsum of the left hand between the bases of the second and third fingers, exposing the extensor expansion at this point. The patient continued with his work after suture and received penicillin intramuscularly for four days. The wuund had healed soundly in seven days.

Case 2.-A trainee cook lacerated the tips of his third and fourth fingers of the left hand, and these required 12 sutures to replace the flaps of skin raised by the cut. The patient continued at work, with his hands frequently in water, yet both wouilds healed in five days.

Case 3.-Excision of the right retro-auricular sebaceous cyst was performed on a trainee clerk and the wound cleanstitched. The wound healed in five days and then required no further dressing.

Case 4.-A ward orderly sustained a V-shaped laceration on the ulnar side of the base of the right fifth finger. Marked haemorrhagic reaction on the first application of nobecutane required use of a firm dry dressing for 24 hours. This was then removed and nobecutane reapplied. The wound healed, with small areas of second-intention healing, in nine days.

\section{Conclusion}

As a dressing for minor surgical and casualty work nobecutane offers many advantages. It is simple to apply and leaves the patient free to continue his work unemcumbered by a bulky dressing that soon becomes moist and dirty. The wound heals quickly, and, apart from the risks of haemorrhage on first application of the plastic dressing, the use of nobecutane appears to be free from complications.

This form of dressing is worthy of further trial in all casualty and minor surgical departments.

I thank Colonel W. R. M. Drew, Officer Commanding, Cambridge Military Hospital, Aldershot, for permission to publish these results, and am grateful for the encouragement received from Lieutenant-Colonel A. B. Dempsey during the trial. I also thank Evans Medical Supplies Limited for a supply of nobecutane.

\section{Medical Memorandum}

\section{"Nobecutane" as a Surgical Dressing}

A paper written by Rob and Eastcott in 1954 stimulated us to use "nobecutane" as a surgical dressing. Since then we have applied this substance to 204 wounds. At first it was used mostly after operations for hernia and appendicitis, but later after major abdominal conditions. We fully endorse the various points of advantage mentioned by the previous writers, but have been particularly impressed by the absence of sepsis. There was infection in only one of the 204 wounds. This occurred in a man of 84 who underwent choledochoduodenostomy : the wound became infected with clostridia, which organisms had been previously cultured from his bile.

It is our impression that the healing of wounds under this application is somewhat better than under the more standard dressings. The slight reddening of the skin along the suture line that is commonly found in healing wounds is usually absent. It is possible that the splinting action of the plastic reduces the small degree of friction and irritation which must occur in the first few days of healing. -

There are various points which we have learned in the technique of its use. Rob and Eastcott advocated the use of a fine layer of the solution applied with a spray. We have had the best results by spreading the solution thickly over the wound with a glass (colostomy) rod. We believe this method is better, because it seals the wound more effectively and to some extent acts as a splint. Another advantage is that when the stitches are to be removed this thick layer can be peeled off in one piece, with the stitches incorporated in it, by simply cutting each stitch in turn as the plastic is raised and removed; this also obviates any necessity to dissolve the plastic with ether or acetone or peel it off in flakes.

The solution comes out of the spray container as an apparently thinner liquid, which runs about more readily and inconveniently than the more viscid fluid in the ordinary bottle which we apply on a glass rod.

It is important that the solution be perfectly dry before the patient leaves the theatre; and when using the thicker layer some patience is required on the part of the operator, although we have found the process appreciably hastened by using a hair-drier. The drier must be at "cold," as if turned to "hot" it tends to liquefy the drying solution. Slightly tacky opposing surfaces of nobecutane tend to stick to each other a little, particularly with midline or paramedian wounds when the patient sits up : this can be prevented by sprinkling the whole area with talcum powder after using the hair-drier.

As the dressing is impermeable to water, patients may be allowed to take a bath on an early post-operative day.

As Rob and Eastcott pointed out, nobecutane should not be applied to a wound in which perfect haemostasis has not been secured. In these cases we have applied the nobecutane in the wards the next day. We have had no adverse criticisms from the patients; indeed, many of them are interested to see the wound through the transparent covering. In young children we have covered the area with a single strip of gauze and strapping after the nobecutane is dry.

We have found nobecutane an ideal dressing for abdominal wounds adjacent to any enterostomy : they remain in perfect condition in the presence of faecal contamination. More nobecutane is applied after the stitches have been removed. We have, however, not found it suitable as a protection to the skin immediately around ileostomies or colostomies, as the faecal matter tends to burrow underneath it.

I should like to thank Mr. J. P. Hosford and Mr. E. G. Tuckwell for allowing me to apply this substance on their patients. Mr. Hosford also helped in the preparation of this paper.

JAMES O. Robinson, M.Chir., F.R.C.S. REFERENCE

Rob, C. G., and Eastcott, H. H. G. (1954). British Medical Journal, 2, 17. 